\title{
Expression and clinical significance of vascular endothelial growth factor and fms-related tyrosine kinase 1 in colorectal cancer
}

\author{
LINCHUN WEN, RUI WANG, XIYAN LU and CHUANWEN YOU \\ Department of Oncology, Suqian People's Hospital of Nanjing Drum Tower Hospital Group, \\ Suqian, Jiangsu 223800, P.R. China
}

Received June 18, 2014; Accepted February 17, 2015

DOI: $10.3892 / 01.2015 .3013$

\begin{abstract}
The aim of the present study was to investigate the prognostic value of vascular endothelial growth factor (VEGF) and its receptor, fms-related tyrosine kinase-1 (FLT-1), in patients with colorectal cancer. An immunohistochemical approach was used to detect the protein expression of VEGF and FLT-1 in 90 patients with colorectal cancer. The impact of VEGF and FLT-1 tumor cell expression, in addition to other factors, on overall survival (OS) was retrospectively assessed in 90 patients. Multivariate analysis was performed in order to determine the prognostic significance of the factors. The positive expression rate of VEGF in the colorectal cancer tissues was $62.2 \%$ (56/90). The positive expression rate of FLT-1 in colorectal cancer tissues was $48.9 \%$ (44/90). The results of the log-rank test revealed that improved OS rates were significantly associated with the absence of VEGF expression $(\mathrm{P}<0.0001)$. By contrast, FLT-1 expression had no significant impact on OS $(\mathrm{P}=0.289)$. Upon multivariate analysis, VEGF expression $(\mathrm{P}=0.038)$ and clinical stage $(\mathrm{P}=0.021)$ maintained significance. VEGF expression proved to be an independent negative predictor of OS in patients with colorectal cancer. Conversely, FLT-1 expression demonstrated no impact on OS.
\end{abstract}

\section{Introduction}

Colorectal cancer is one of the most frequently diagnosed malignant diseases, with an estimated 1,023,000 new cases and 529,000 associated mortalities each year worldwide (1). As a result of improved living standards and changes in eating habits, the incidence of colorectal cancer in China has increased, and it is now ranked as the fifth most lethal malignancy. Despite improvements in treatment strategies, patients

Correspondence to: Mr. Linchun Wen, Department of Oncology, Suqian People's Hospital of Nanjing Drum Tower Hospital Group, 138 Huanghe South Road, Suqian, Jiangsu 223800, P.R. China E-mail: wenlinchun@126.com

Key words: colorectal cancer, vascular endothelial growth factor, fms-like tyrosine kinase-1, immunohistochemistry, prognosis with colorectal cancer have a relatively poor prognosis. Therefore, the identification of optimal prognostic factors and the development of personalized treatments is extremely important. Previous studies have demonstrated that the mutational status of the K-ras gene is important for the treatment of advanced colorectal cancer with cetuximab, as it affects the tumor response and has treatment-independent prognostic value $(2,3)$.

Vascular endothelial growth factor (VEGF) is a diffusible glycoprotein produced by normal and neoplastic cells, which regulates physiological and pathological angiogenesis $(4,5)$. Tumor development is a complex biological process that involves a number of genes. Previous studies (6-10) have demonstrated that angiogenesis is closely associated with the formation, development and prognosis of malignant tumors, in which VEGF and VEGF receptor-1 (VEFGR-1), also known as fms-like tyrosine kinase-1 (FLT-1), are the core regulating factors. The prognostic value of the tumor cell expression of VEGF and its receptor, FLT-1, remains controversial. VEGF has been reported to be associated with the clinical outcomes of a number of tumors, including head and neck cancer, esophageal cancer and thyroid carcinoma (10-13). By contrast, a similar correlation was not shown for pancreatic adenocarcinoma, epithelial ovarian cancer or non-small cell lung cancer by other studies $(6,14,15)$. Therefore, further investigation is required in order to better define the predictive value of these two potential prognostic factors in colorectal cancer. The present study evaluated the expression of VEGF and FLT-1, and their correlation with clinicopathological factors and clinical outcomes, in patients with colorectal cancer.

\section{Materials and methods}

Materials. In total, 90 paraffin samples with complete clinical data obtained from primary colorectal cancer patients who had undergone surgery at the Suqian People's Hospital of Nanjing Drum Tower Hospital Group (Suqian, Jiangsu, China) between January 2007 and June 2009 were eligible for use in the present study. The study was approved by the Ethics Committee of Suqian People's Hospital of Nanjing Drum Tower Hospital and written informed consent was obtained from all patients. In total, 90 patients, including 55 males and 35 females, aged between 37 and 81 years old, with a median age of 63.8 years, 
were retrospectively analyzed. The additional patient characteristics are summarized in Table I. The primary tumor sites were as follows: i) ileocecal back, 6 cases; ii) ascending colon, 20 cases; iii) transverse colon, 7 cases; iv) descending colon, 13 cases; v) sigmoid colon, 11 cases; and vi) rectum, 33 cases. Overall, lymph node metastases were present in 39 cases, and absent in 51 cases. Dukes' staging was recorded as follows: i) A, 8 cases; ii) B, 22 cases; iii) C, 49 cases; and iv) D, 11 cases. According to the World Health Organization colorectal adenocarcinoma differentiation standards, there were 38 highly-differentiated cases, 31 median-differentiated cases and 21 poorly-differentiated cases (16).

Immunohistochemistry examination. The archived paraffin-embedded tissues were used to generate consecutive $4-\mu \mathrm{m}$ thick sections. The streptavidin-biotin complex (sABC) method with a known positive colorectal biopsy was used as a positive control, and phosphate buffered saline was used as a negative control. The mouse anti-human VEGF monoclonal antibody (mAb; 1:100), mouse anti-human FLT-1 mAb (1:100), a universal quick method secondary antibody, and the diaminobenzidine (DAB) chromogenic kit were all purchased from Beijing Zhongshan Golden Bridge Biotechnology Co., Ltd. (Beijing, China).

The staining procedure was as follows: The slices were dewaxed, followed by application of $30 \mathrm{ml} / 1 \mathrm{H}_{2} \mathrm{O}_{2}$ methanol solution to block endogenous peroxidase activity and the addition of digestive juices to digest the tissues. Next, the secondary antibody and sABC reagents were applied, as well as 3-amino-9-ethylcarbazole color. The slides were then stained with hematoxylin and dehydrated in a graded alcohol series, followed by the addition of xylene and neutral gum cementing. The slides were then assessed under a light microscope using a double-blind format (AX70, Olympus Corporation, Tokyo, Japan). Red staining in the nucleus or cytoplasm was used to indicate a positive result.

VEGF and FLT-1 proteins. VEGF and FLT-1 staining was determined as follows: i) Positive, $\geq 10 \%$ of the cancer cells stained; and ii) negative, no positive staining or $<10 \%$ of the cancer cells stained.

Statistical analysis. The associations between VEGF and FLT-1 expression and the clinicopathological parameters were statistically analyzed by $\chi^{2}$ test using SPSS version 13.0 software (SPSS, Inc., Chicago, IL, USA). The overall survival (OS) rates were determined using the Kaplan-Meier method and compared by the log-rank test. Multivariate analysis for survival was performed using a Cox proportional hazards regression model. $\mathrm{P}<0.05$ was used to indicate a statistically significant difference.

\section{Results}

Expression of VEGF and FLT-1 in colorectal cancer. VEGF expression was evident in the cytoplasm and cell membranes. The overall positive expression rate was $62.2 \%(56 / 90)$. FLT-1 was only observed in the cytoplasm of primarily tumor vascular endothelial cells, with a positive expression rate of $48.9 \%(44 / 90)$.

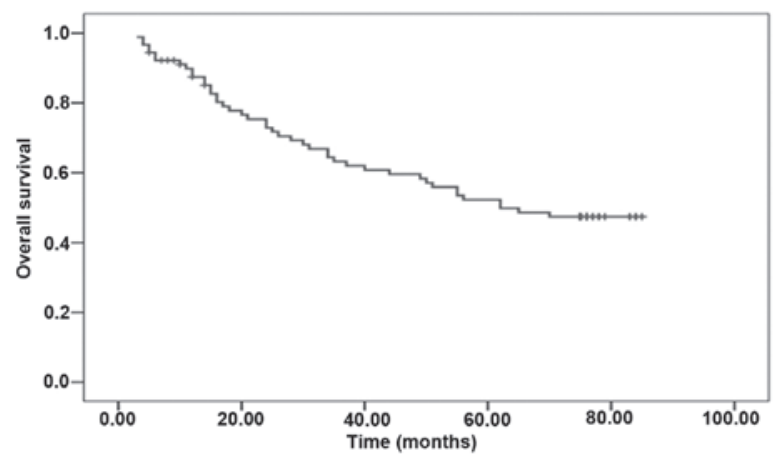

Figure 1. Overall survival curve of ovarian carcinoma patients revealing a median survival time of 62.00 months.

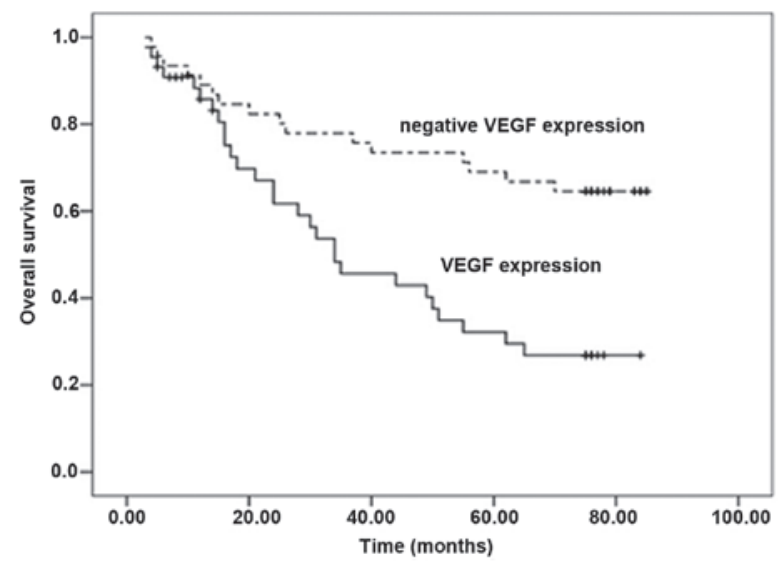

Figure 2. Impact of VEGF expression on the overall survival of patients with colorectal cancer $\left(\chi^{2}=13.501, \mathrm{P}<0.0001\right)$.

Association between VEGF and FLT-1 expression and clinicopathological factors. VEGF expression was associated with the histological grade, depth of invasion, lymph node metastasis and Dukes' stage of the colorectal cancer $(\mathrm{P}<0.05)$. FLT-1 expression was associated with the depth of invasion and lymph node metastasis $(\mathrm{P}<0.05)$. Specific correlations between VEGF and FLT-1 and age, gender, degree of differentiation, depth of invasion, lymph node metastasis and Dukes' staging are shown in Table I.

Prognostic value of VEGF and FLT-1 expression in colorectal cancer. In order to investigate the prognostic value of VEGF and FLT-1 expression in colorectal cancer, cumulative survival curves for the 90 patients with colorectal cancer were constructed according to the Kaplan-Meier method. Differences in OS were then assessed using the log-rank test. The median OS time of the 90 patients was 62.00 months (Fig. 1). Upon univariate analysis, the OS rates of the colorectal cancer patients with VEGF expression were significantly reduced compared with the patients with no VEGF expression $(\mathrm{P}<0.0001$; Fig. 2). Improved OS was significantly associated with negative VEGF expression. By contrast, a trend toward improved OS was observed for negative FLT-1 expression ( $\mathrm{P}=0.289$; Fig. 3 ).

Following multivariate Cox proportional hazards regression analysis, VEGF expression and Dukes' stage were 
Table I. Association between VEGF and FLT-1 expression and the clinicopathological characteristics of colorectal cancer ( $\mathrm{n}=90)$.

\begin{tabular}{|c|c|c|c|c|c|c|c|c|c|}
\hline \multirow[b]{2}{*}{ Groups } & \multirow[b]{2}{*}{$\mathrm{n}$} & \multicolumn{4}{|c|}{ VEGF } & \multicolumn{4}{|c|}{ FLT-1 } \\
\hline & & - & + & $\chi^{2}$ & P-value & - & + & $\chi^{2}$ & $\mathrm{P}$-value \\
\hline Age, years & & & & 2.054 & 0.152 & & & 1.558 & 0.212 \\
\hline$<60$ & 51 & 16 & 35 & & & 29 & 22 & & \\
\hline$\geq 60$ & 39 & 18 & 21 & & & 17 & 22 & & \\
\hline Gender & & & & 0.010 & 0.921 & & & 1.800 & 0.178 \\
\hline Male & 55 & 21 & 34 & & & 25 & 30 & & \\
\hline Female & 35 & 13 & 22 & & & 21 & 14 & & \\
\hline Histological grade & & & & 14.546 & 0.001 & & & 3.824 & 0.148 \\
\hline Well & 38 & 23 & 15 & & & 24 & 14 & & \\
\hline Moderately & 31 & 7 & 24 & & & 13 & 18 & & \\
\hline Poorly & 21 & 4 & 17 & & & 9 & 12 & & \\
\hline Depth of invasion & & & & 4.618 & 0.032 & & & 4.211 & 0.040 \\
\hline $\mathrm{T} 1, \mathrm{~T} 2$ & 23 & 13 & 10 & & & 16 & 7 & & \\
\hline $\mathrm{T} 3, \mathrm{~T} 4$ & 67 & 21 & 46 & & & 30 & 37 & & \\
\hline Lymph node metastasis & & & & 6.328 & 0.012 & & & 6.375 & 0.012 \\
\hline Negative & 51 & 25 & 26 & & & 32 & 19 & & \\
\hline Positive & 39 & 9 & 30 & & & 14 & 25 & & \\
\hline Dukes' stage & & & & 29.423 & 0.000 & & & 2.012 & 0.570 \\
\hline A & 8 & 6 & 2 & & & 4 & 4 & & \\
\hline B & 22 & 17 & 5 & & & 13 & 9 & & \\
\hline $\mathrm{C}$ & 49 & 8 & 41 & & & 22 & 27 & & \\
\hline $\mathrm{D}$ & 11 & 3 & 8 & & & 7 & 4 & & \\
\hline
\end{tabular}

VEGF, vascular endothelial growth factor; FLT-1, fms-related tyrosine kinase-1.

Table II. Multivariate Cox proportional hazards regression analysis.

\begin{tabular}{lcc}
\hline Variable & Wald $\chi^{2}$ & P-value \\
\hline VEGF & 4.316 & 0.038 \\
FLT-1 & 3.655 & 0.056 \\
Dukes' stage & 5.314 & 0.021 \\
Lymph node metastasis & 1.154 & 0.283 \\
Depth of invasion & 2.461 & 0.117 \\
Histological grade & 0.531 & 0.466 \\
\hline
\end{tabular}

VEGF, vascular endothelial growth factor; FLT-1, fms-related tyrosine kinase-1.

revealed to be significant independent prognostic indicators of OS ( $\mathrm{P}=0.038$ and $\mathrm{P}=0.021$, respectively; Table II).

\section{Discussion}

Angiogenesis results in the formation of new blood vessels from a pre-existing vascular network, and is therefore required for tumor growth, invasion and metastasis. VEGF was first

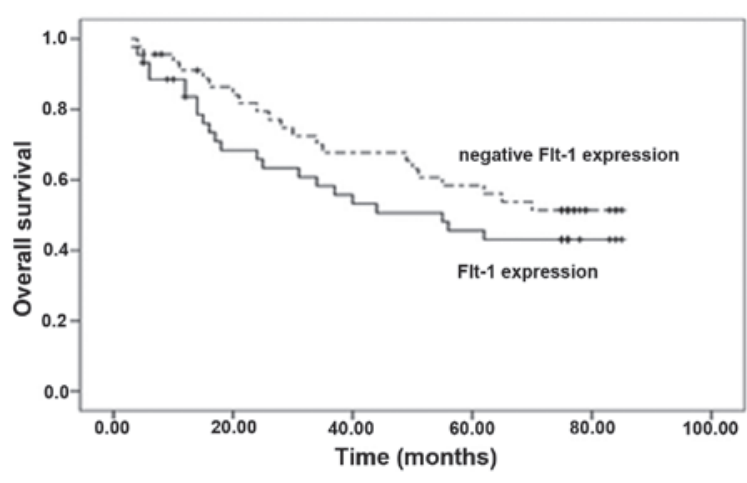

Figure 3. Impact of FLT-1 expression on the overall survival of patients with colorectal cancer $\left(\chi^{2}=1.125, \mathrm{P}=0.289\right)$. FLT-1, fms-related tyrosine kinase- 1 .

purified from the culture medium of bovine pituitary follicular stellate cells in 1989 by Bellamy et al (7), and was later identified to be a homologous glycoprotein with heparin-binding activity. VEGF is an endothelial cell-specific mitogen promoter (17). As a potent growth factor acting directly on vascular endothelial cells, VEGF has an important role in the division, proliferation and migration of vascular endothelial cells. Synthesized and released by endothelial cells, granulocytes and megakaryocytes, VEGF is widely present in body 
tissues, and participates in the progression of a variety of diseases (18). As a vascular inducing factor in angiogenesis, VEGF can induce and enhance the permeability of blood vessels. In addition, it aids in the maintenance of the normal state and integrity of blood vessels. VEGFRs are extensively expressed in vascular endothelial cells. VEGF binds with specific receptors in order to exert its biological functions; in particular, increasing microvascular growth and permeability (19). VEGFR-1, also known as FLT-1, is primarily located on vascular endothelial cells, and is expressed, albeit to a lesser degree, on mononuclear cells, mesangial cells and trophoblast cells. VEGF exerts its functions by binding to and activating FLT-1, stimulating the proliferation of vascular endothelial cells, increasing vascular permeability and promoting the formation of new blood vessels. Therefore, VEGF and VEGFR-1 are considered to be the most promising anti-angiogenic targets (20).

VEGF plays an important role in angiogenesis by activating FLT-1 and KDR to stimulate the growth of tumor vascular endothelial cells. Boiocchi et al (21) revealed that, in hepatocellular carcinoma, angiogenesis is primarily mediated by the VEGF/FLT-1 system. Furthermore, in angiogenesis-rich regions, the incidence and sensitivity of apoptosis is reduced. VEGFRs are involved in the complex proliferation interaction between gastric cancer cells and endothelial cells. In addition, it has been demonstrated that FLT-1 cannot reverse the tumor angiogenesis inhibition caused by negative KDR mutations, which indicates that FLT-1 may mediate the non-mitogenic effects caused by VEGF, and not the mitogenic response $(9,22,23)$.

However, despite these results, the prognostic value of the tumor cell expression of VEGF and FLT-1 requires further investigation, as available data are currently inconclusive. Several studies $(14,24,25)$ have confirmed that VEGF expression is negatively associated with patient prognosis in esophageal, and head and neck cancers. By contrast, other studies have revealed no significant prognostic association in patients with non-small cell lung and epithelial ovarian cancer. Nevertheless, the majority of studies consistently illustrate that VEGF expression is negatively corrected with patient prognosis. With regard to the prognostic value of FLT-1 expression, available data in the literature are even more heterogeneous than those for VEGF expression. Certain studies $(6,11,14)$ have suggested a negative prognostic value of FLT-1 expression on the clinical outcome of thyroid carcinomas or non-small cell lung cancers, while others have demonstrated no significant correlation between FLT-1 expression and the outcome of esophageal or ovarian cancers $(12,15)$. Furthermore, two previous studies $(6,26)$ even established a positive impact of FLT-1 expression on survival in pancreatic or locally advanced breast cancer.

The results of the present study revealed positive expression rates of VEGF at $62.2 \%(56 / 90)$ and FLT-1 at $48.9 \%$ (44/90) in 90 cases of colorectal cancer. The positive expression rates of VEGF and FLT-1 in the present study were slightly lower than those reported in previous studies, which may be due to the earlier clinical stages and advanced age of the patients included in the present study. It was demonstrated that VEGF expression was significantly negatively associated with OS in colorectal cancer patients, which is consistent with the results of previous studies (20,27-29). Seibord et al (29) reported poorer survival rates in patients with VEGF-positive tumors in a retrospective series of 117 patients. Furthermore, Kato et al (12) demonstrated that VEGF expression was associated with a poor prognosis in a retrospective series of 64 patients (12). The results of the present study indicate that VEGF has an important role in the proliferation, invasion and metastasis of colorectal cancer. However, the utility of FLT-1 as a prognostic indicator in colorectal cancer remains unclear, as the present study failed to demonstrate prognostic value. This result is similar to those of previous retrospective studies that did not identify a correlation between FLT-1 expression and the treatment outcomes of various tumor types, including epithelial ovarian (15), tongue (13), nasopharyngeal (30) and non-small cell lung (31) cancer. However, two other retrospective studies $(6,26)$ suggested that FLT-1 expression was associated with favorable outcomes. Therefore, according to the present dilemma regarding FLT-1, it can be hypothesized that the role of FLT-1 in tumor growth and spread is likely to be diverse and complex.

In conclusion, in the present study, VEGF expression proved to be an independent negative predictor of OS in patients with colorectal cancer, whereas FLT-1 expression appeared to have no significant impact on clinical outcome. Owing to the limitations of the retrospective study design, these results should be confirmed by prospective studies.

\section{References}

1. Saltz LB, Cox JV, Blanke C, et al: Irinotecan plus fluorouracil and leucovorin for metastatic colorectal cancer. N Engl J Med 343: 905-914, 2000.

2. Imamura Y, Lochhead P, Yamauchi M, et al: Analyses of clinicopathological, molecular and prognostic associations of KRAS codon 61 and codon 146 mutations in colorectal cancer: cohort study and literature review. Mol Cancer 13: 135, 2014.

3. Buchler T, Pavlik T, Melichar B, et al: Bevacizumab with 5-fluorouracil, leucovorin and oxaliplatin versus bevacizumab with capecitabine and oxaliplatin for metastatic colorectal carcinoma: results of a large registry-based cohort analysis. BMC Cancer 14: 323, 2014.

4. Takahashi S: Vascular endothelial growth factor (VEGF), VEGF receptors and their inhibitors for antiangiogenic tumor therapy. Biol Pharm Bull 34: 1785-1788, 2011.

5. Saif MW: Antiangiogenesis therapy in second line metastatic colorectal cancer: similar but different. Expert Opin Biol Ther 13: 1489-1493, 2013.

6. Chung GG, Yoon HH, Zerkowski MP, et al: Vascular endothelial growth factor, FLT-1 and FLK-1 analysis in a pancreatic cancer tissue microarray. Cancer 106: 1677-1684, 2006.

7. Bellamy WT, Richter L, Frutiger Y and Grogan TM: Expression of vascular endothelial growth factor and its receptors in hematopoietic malignancies. Cancer Res 59: 728-733, 1999.

8. Daenen LG, Roodhart JM, van Amersfoort M, etal: Chemotherapy enhances metastasis formation via VEGFR-1-expressing endothelial cells. Cancer Res 71: 6976-6985, 2011.

9. Lee YJ, Karl DL, Maduekwe UN, et al: Differential effects of VEGFR-1 and VEGFR-2 inhibition on tumor metastases based on host organ environment. Cancer Res 70: 8357-8367, 2010.

10. Takahashi Y, Kitadai Y, Bucana CD, Cleary KR and Ellis LM: Expression of vascular endothelial growth factor and its receptor, KDR, correlates with vascularity, metastasis and proliferation of human colon cancer. Cancer Res 55: 3964-3968, 1995.

11. Fenton C, Patel A, Dinauer C, Robie DK, Tuttle RM and Francis GL: The expression of vascular endothelial growth factor and the type 1 vascular endothelial growth factor receptor correlate with the size of papillary thyroid carcinoma in children and young adults. Thyroid 10: 349-357, 2000 
12. Kato H, Yoshikawa M, Miyazaki T, et al: Expression of vascular endothelial growth factor (VEGF) and its receptors (Flt-1 and Flk-1) in esophageal squamous cell carcinoma. Anticancer Res 22: 3977-3984, 2002

13. Tanigaki Y, Nagashima Y, Kitamura Y, Matsuda H, Mikami Y and Tsukuda M: The expression of vascular endothelial growth factor- $\mathrm{A}$ and $-\mathrm{C}$ and receptors 1 and 3: correlation with lymph node metastasis and prognosis in tongue squamous cell carcinoma. Int J Mol Med 14: 389-395, 2004.

14. Dales JP, Garcia S, Bonnier P, et al: Prognostic significance of VEGF receptors, VEGFR-1 (Flt-1) and VEGFR-2 (KDR/Flk-1) in breast carcinoma. Ann Pathol 23: 297-305, 2003 (In French).

15. Secord AA, Darcy KM, Hutson A, et al; Gynecologic Oncology Group study: Co-expression of angiogenic markers and associations with prognosis in advanced epithelial ovarian cancer: a Gynecologic Oncology Group study. Gynecol Oncol 106: 221-232, 2007.

16. Hamilton SR and Aaltonen LA (eds.): World Health Organization Classification of Tumours. Pathology and Genetics of Tumors of the Digestive System. IARC Press, Lyon, France, 2000.

17. Itakura J, Ishiwata $\mathrm{T}$, Shen B, Kornmann $\mathrm{M}$ and Korc $\mathrm{M}$ : Concomitant over-expression of vascular endothelial growth factor and its receptors in pancreatic cancer. Int J Cancer 85: 27-34, 2000

18. Delmotte P, Martin B, Paesmans M, et al: VEGF and survival of patients with lung cancer: a systematic literature review and meta-analysis. Rev Mal Respir 19: 577-584, 2002 (In French).

19. Bando H, Weich HA, Brokelmann M, et al: Association between intratumoral free and total VEGF, soluble VEGFR-1, VEGFR-2 and prognosis in breast cancer. Br J Cancer 92: 553-561, 2005.

20. Wang L, Liu X, Wang H and Wang S: Correlation of the expression of vascular endothelial growth factor and its receptors with microvessel density in ovarian cancer. Oncol Lett 6: 175-180, 2013.

21. Boiocchi L, Vener C, Savi F, et al: Increased expression of vascular endothelial growth factor receptor 1 correlates with VEGF and microvessel density in Philadelphia chromosome-negative myeloproliferative neoplasms. J Clin Pathol 64: 226-231, 2011.

22. Okita NT, Yamada Y, Takahari D, et al: Vascular endothelial growth factor receptor expression as a prognostic marker for survival in colorectal cancer. Jpn J Clin Oncol 39: 595-600, 2009.
23. Zhan P, Wang J, Lv XJ, et al: Prognostic value of vascular endothelial growth factor expression in patients with lung cancer: a systematic review with meta-analysis. J Thorac Oncol 4: 1094-1103, 2009.

24. Chen G, Ding J, Luo L, et al: Expression of vascular endothelial growth factor and its receptors in laryngeal carcinoma cell and its significance. Lin Chuang Er Bi Yan Hou Ke Za Zhi 19: 842-844, 2005 (In Chinese).

25. Fan F, Wey JS, McCarty MF, et al: Expression and function of vascular endothelial growth factor receptor-1 on human colorectal cancer cells. Oncogene 24: 2647-2653, 2005.

26. Zhukova LG, Zhukov NV and Lichinitser MR: Expression of Flt-1 and Flk-1 receptors for vascular endothelial growth factor on tumor cells as a new prognostic criterion for locally advanced breast cancer. Bull Exp Biol Med 135: 478-481, 2003.

27. Dhakal HP, Naume B, Synnestvedt M, et al: Expression of vascular endothelial growth factor and vascular endothelial growth factor receptors 1 and 2 in invasive breast carcinoma: prognostic significance and relationship with markers for aggressiveness. Histopathology 61: 350-364, 2012.

28. Kopparapu PK, Boorjian SA, Robinson BD, et al: Expression of VEGF and its receptors VEGFR1/VEGFR2 is associated with invasiveness of bladder cancer. Anticancer Res 33: 2381-2390, 2013.

29. Seibold ND, Schild SE, Bruchhage KL, Gebhard MP, Noack F and Rades D: Prognostic impact of VEGF and FLT-1 receptor expression in patients with locally advanced squamous cell carcinoma of the head and neck. Strahlenther Onkol 189: 639-646, 2013.

30. Sha D and He YJ: Expression and clinical significance of VEGF and its receptors Flt-1 and KDR in nasopharyngeal carcinoma. Ai Zheng 25: 229-234, 2006 (In Chinese)

31. Rades D, Setter C, Dunst J, Dahl O, Schild SE and Noack F: Prognostic impact of VEGF and VEGF receptor 1 (FLT1) expression in patients irradiated for stage II/III non-small cell lung cancer (NSCLC). Strahlenther Onkol 186: 307-314, 2010. 